\title{
What Can a False-Negative Transcranial Motor Evoked Potential Envisage the Outcome in Isolated Position-Related Praxis?
}

\author{
Vijayashree $\mathrm{T}^{1}$ \\ Dheeraj Masapu ${ }^{1}$ \\ Deepak Rajappa \\ ${ }^{1}$ Department of Anaesthesia, Sakra World Hospital, Bellandur, \\ Karnataka, India \\ 2Department of Neurosciences, Sakra World Hospital, Bellandur, \\ Karnataka, India
}

J Neuroanaesthesiol Crit Care 2021;8:230-231.

Surgical procedures involving the spine require prone positioning and are not devoid of complications. Perioperative peripheral nerve injury (PPNI) is an important complication with an incidence of 0.03 to $0.1 \%$ and with $0.02 \%$ incidence of brachial plexopathy in noncardiac surgery. ${ }^{1}$ Intraoperative neuromonitoring (IONM) used during spine surgery detects peripheral nerve conduction abnormalities, thus preventing PPNI. ${ }^{1}$ Here we report a case of false-negative transcranial motor evoked potential (TcMEP) leading to transient right upper limb weakness postoperatively.

A 50-year-old woman presented with back pain and difficulty in walking for 3 months. On examination, sensory deficit was noted below umbilicus. The motor power was $4 / 5$ in both lower limbs according to Medical Research Council (MRC) grading. Magnetic resonance imaging (MRI) spine revealed intradural extramedullary (IDEM) tumor at thoracic vertebra level 7-8 (D7-D8) pushing the spinal cord to left side with significant cord compression; D7-8 laminectomy and excision of the tumor was planned. Anesthesia was induced with propofol $1.5 \mathrm{mg} / \mathrm{kg}$ and fentanyl $2 \mu \mathrm{g} / \mathrm{kg}$, and maintained with total intravenous anesthesia using propofol $150 \mu \mathrm{g} / \mathrm{kg} / \mathrm{min}$ and fentanyl $1 \mu \mathrm{g} / \mathrm{kg} / \mathrm{h}$ infusions. Muscle relaxants were avoided. TcMEP were recorded with electrodes inserted in scalp at C3 and C4 (international10-20 system). Compound muscle action potentials (CMAPs) were recorded from right abductor digiti minimi in the upper limbs as reference, and tibialis anterior and adductor hallucis longus in the bilateral lower limbs. The impedance of all electrodes was maintained below $5 \mathrm{kV}$. Patient was placed in the prone surrender (superman) position ${ }^{1}$ for a total surgical duration of 3 hours. Superman/surrender prone position involves the patient to be placed prone with head in neutral position, shoulders abducted to less than $90^{\circ}$, lateral rotation of upper arms and published online August 8, 2021
DOI https://doi.org/ $10.1055 / \mathrm{s}-0041-1730097$ ISSN 2348-0548

\author{
Satish Rudrappa ${ }^{2} \quad$ N. Chandrashekar ${ }^{2}$
}

Address for correspondence: Dheeraj Masapu, MD, DM, Department of Anaesthesia, Sy. No. 52/2 and 52/3, Devarabeesanahalli, Varthur Hobli, Sakra World Hospital, Opposite Intel, Outer Ring Road, Marathahalli, Bellandur, Karnataka 560103, India (e-mail: dheerajmasapu@gmail.com).

elbows flexed and positioned in neutral position at or below the mattress level beside the patient's head. Normothermia and normocapnia were maintained during the procedure with a total blood loss of $150 \mathrm{~mL}$. A significant drop in power was noted in the right upper limb involving the cervical (C) 5,6,7 nerve root distribution affecting the muscles like deltoid, biceps, triceps, and brachioradialis, with MRC grading of $2 / 5$ up on extubation. The intrinsic muscles of the arm which are innervated by the ulnar nerve were spared. Baseline power was maintained in rest of the limbs. However, no change in amplitude in TcMEPs was detected during the procedure ( - Fig. 1 ).

Possible differential diagnosis of upper limb weakness include cervical disc compression, stroke transient ischemic attack (TIA), vertebral artery dissection or positional brachial plexopathy. ${ }^{1,2}$ Postoperative MRI scan did not reveal any signs of stroke, vertebral artery dissection, and cord compression. After all the other causes were ruled out, diagnosis of unilateral positional brachial plexopathy (neuropraxia) was made. The patient was reassured about the recovery of the limb function and complete recovery to baseline power did take three days.

Mechanism of PPNI includes ischemia of nerve fibers due to stretch/compression and direct trauma. Risk factors for position related brachial plexus injuries include use of shoulder braces and head-down position, shoulder abduction greater than $90^{\circ}$, external rotation of the arm, and posterior shoulder displacement. ${ }^{1}$ IONM plays a crucial role in detecting PPNI as demonstrated by Luginbuhl et al. ${ }^{3}$ The arm was returned to neutral position after evolving plexopathy was detected with IONM. Tsutsui et al ${ }^{4}$ evaluated the effect of spinal cord or root lesioning on 5-pulse TES MEPs from the hind limb triceps surae muscle in cats and found that CMAPs decreased

(c) 2021. Indian Society of Neuroanaesthesiology and Critical Care. This is an open access article published by Thieme under the terms of the Creative Commons Attribution-NonDerivative-NonCommercial-License, permitting copying and reproduction so long as the original work is given appropriate credit. Contents may not be used for commercial purposes, or adapted, remixed, transformed or built upon. (https://creativecommons.org/licenses/by-nc-nd/4.0/).

Thieme Medical and Scientific Publishers Pvt. Ltd. A-12, 2nd Floor, Sector 2, Noida-201301 UP, India 


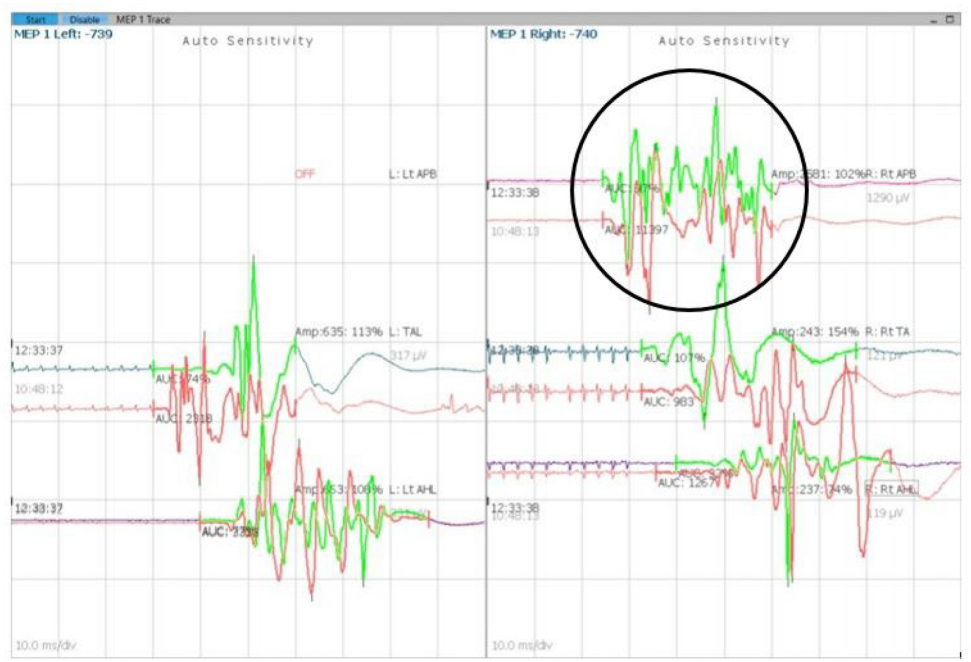

Fig. 1 Encircled image showing no change in the transcranial motor evoked potentials (TcMEPs) in the affected right upper limb.

in amplitude significantly after the insult to the corticospinal tracts in the spinal cord in all cats, but the potentials did not always change when the insult was restricted to a limited area in the anterior horn of the spinal cord or to the single spinal nerve roots. This might explain the reason for the false-negative findings in our case. Similar false-negative case was reported by Modi et $\mathrm{al}^{5}$ where the patient developed paraplegia with intact TcMEP. Another possible explanation is that the TCMEP was recorded from the intrinsic muscles of the hand which are supplied by the ulnar nerve $(C 8, T 1)$ while the actual loss of power happened in the shoulder abduction which is innervated by the $C 5,6,7$ nerve roots.

Limitations of our case includes inability to perform nerve conduction studies, which would have showed slowing of conduction time ${ }^{6}$ it would add value in differentiating between the affected nerve roots and nonaffected nerve roots, and it can help in assessing the progress of the condition. Also, lack of somatosensory evoked potential (SSEP) monitoring, which would have picked up the praxia in the early stages. ${ }^{7}$

We conclude that TCMEP, although considered paragon, may not be a foolproof method to negate postoperative motor deficit, but it can play a significant role in reassuring the patient regarding the motor recovery. Addition of other modalities like somatosensory evoked potential and nerve conduction studies would add more value and information in prognostication of the motor recovery.
Conflict of Interest

None declared.

\section{References}

1 Kamel I, Barnette R. Positioning patients for spine surgery: avoiding uncommon position-related complications. World J Orthop 2014;5(4):425-443

2 Chowdhury T, Goyal K, Sapra H, Mehta Y. Upper limb weakness following lumber disc surgery: an unusual case. Saudi J Anaesth 2012;6(3):302-303

3 Luginbuhl A, Schwartz DM, Sestokas AK, Cognetti D, Pribitkin E. Detection of evolving injury to the brachial plexus during transaxillary robotic thyroidectomy. Laryngoscope 2012;122(1):110-115

4 Tsutsui S, Tamaki T, Yamada $\mathrm{H}$, Iwasaki $\mathrm{H}$, Takami M. Relationships between the changes in compound muscle action potentials and selective injuries to the spinal cord and spinal nerve roots. Clin Neurophysiol 2003;114(8):1431-1436

5 Modi HN, Suh SW, Yang JH, Yoon JY. False-negative transcranial motor-evoked potentials during scoliosis surgery causing paralysis: a case report with literature review. Spine 2009;34(24):E896-E900

6 Robinson LR. Traumatic injury to peripheral nerves. Muscle Nerve 2000;23(6):863-873

7 Jellish WS, Sherazee G, Patel J, et al. Somatosensory evoked potentials help prevent positioning-related brachial plexus injury during skull base surgery. Otolaryngol Head Neck Surg 2013;149(1):168-173 